\title{
Long term follow up of prosthetic valve endocarditis: what characteristics identify patients who were treated successfully with antibiotics alone?
}

\author{
K Truninger, C H Attenhofer Jost, B Seifert, P R Vogt, F Follath, A Schaffner, R Jenni
}

\begin{abstract}
Objective-To identify predictors for the safe use of antibiotic treatment without reoperation in patients with prosthetic valve endocarditis.

Setting-Retrospective study in a tertiary care centre.

Subjects and design-All 49 episodes of definite prosthetic valve endocarditis (Duke criteria) diagnosed at one institution between 1980 to 1997 were analysed. Ten episodes $(20 \%)$ were treated with antibiotics only (antibiotic group) and 39 episodes $(80 \%)$ with combined antibiotic and surgical treatment (surgery group). The analysis included detailed study of hospital records and data on long term follow up which were obtained in all patients by a questionnaire or telephone contact with physician or patient. The length of follow up (mean (SD)) was 41 (32) months in the antibiotic group and 45 (24) months in the surgery group (NS). Long term survival was estimated by the Kaplan-Meier method and compared by the log-rank test.
\end{abstract}

Department of Internal Medicine, University Hospital Zurich, Raemistrasse 100, 8091 Zurich, Switzerland K Truninger F Follath

A Schaffner

Division of Cardiology, University Hospital Zurich

C H Attenhofer Jost R Jenni

Clinic for Cardiovascular Surgery, University Hospital Zurich P R Vogt

Department of Biostatistics, ISPM, University of Zurich, Sumatrastrasse 30, 8006 Zurich,

Switzerland

B Seifert

Correspondence to: Dr Jenni.

email: karjer@usz.unizh

Accepted for publication 21 June 1999 nificant difference between the surgery and antibiotic groups regarding late mortality $(14 \% v 18 \%)$ and five year rates of recurrent endocarditis $(14 \% v 16 \%)$, event related mortality (14\% v 3\%, log-rank test), and the need for reoperation (14\% v 19\%; log-rank test). The only patient with conservatively treated staphylococcal prosthetic valve endocarditis died after reoperation for recurrence.

Conclusions-Haemodynamically stable patients with non-staphylococcal prosthetic valve endocarditis who are carefully supervised can be treated with antibiotics alone without an increased rate of reinfection, reoperation, or death.

(Heart 1999;82:714-720)

Keywords: prosthetic valve endocarditis; antibiotic treatment; complications; long term follow up

Prosthetic valve endocarditis is a rare but serious complication after heart valve replacement, with an incidence of approximately $0.1-4 \%$ per year. ${ }^{1-8}$ This type of endocarditis is associated with high morbidity and mortality rates of between $10 \%$ and $59 \% .^{157}$ Generally, combined medical and surgical treatment is considered the treatment of choice and thought to be necessary to improve prognosis. Some clinicians, however, recommend reoperation only in the presence of large vegetations with thromboembolic episodes, congestive heart failure, valve dysfunction, and more virulent organisms. $^{59}$ In a multicentre, prospective, unrandomised trial, however, mortality after valve replacement was lower than after medical treatment alone $(23 \% v 56 \%)^{10}$; the authors of that paper recommend early surgery. Another argument for early surgery was the finding of persistently positive bacterial cultures from prosthetic valves removed at operation or necropsy, despite prolonged bactericidal antibiotic treatment in another study. ${ }^{11}$ Early total excision of the infected annular root was therefore recommended. ${ }^{11}$ Thus controversy continues about when conservative treatment alone can be safe. There are few data analysing subgroups of patients who can be managed relatively safely with medical treatment alone, with an outcome comparable to that achieved with combined surgical and medical treatment.

Our aim in this study was therefore to analyse data from patients with definite prosthetic valve endocarditis to determine predictors identifying those in whom medical treatment alone may be safe.

\section{Methods}

PATIENTS

From January 1980 to September 1997, 49 consecutive patients with definite prosthetic valve endocarditis were admitted to hospital at the University Hospital, Zurich. Three died immediately after admission before treatment decisions could be made and were thus excluded from the study. The remaining 46 patients (nine women, 37 men) had 49 episodes of endocarditis, which are the subject 
Table 1 Clinical characteristics in the two patient groups

\begin{tabular}{|c|c|c|c|}
\hline & $\begin{array}{l}\text { Surgery group } \\
(n=39)\end{array}$ & $\begin{array}{l}\text { Antibiotic group } \\
(n=10)\end{array}$ & $p$ Value \\
\hline Age (years) (mean (SD)) & $50(15)$ & $59(10)$ & NS \\
\hline Male sex & 33 & 8 & NS \\
\hline Concomitant heart disease $^{\star}$ & 29 & 5 & NS \\
\hline \multicolumn{4}{|l|}{ Previous heart operations } \\
\hline One & 21 & 2 & \\
\hline Two & 14 & 6 & \\
\hline Three & 4 & 2 & NS \\
\hline Previous endocarditis & 15 & 2 & NS \\
\hline \multicolumn{4}{|l|}{ Onset of endocarditis } \\
\hline Early ( $\leqslant 2$ months) & 4 & 2 & \\
\hline Late ( $>2$ months) & 35 & 8 & NS \\
\hline Heart failure & 9 & 0 & NS \\
\hline Need for catecholamines & 9 & 0 & NS \\
\hline Embolic events & 16 & 5 & NS \\
\hline \multicolumn{4}{|l|}{ Duration of antibiotic treatment (days) } \\
\hline (mean (SD)) & $41(17)$ & $102(94)$ & 0.039 \\
\hline
\end{tabular}

${ }^{\star}$ Concomitant heart disease was defined as coronary artery disease, hypertensive heart disease, hypertrophic obstructive cardiomyopathy.

of this analysis. Ten of these were treated with antibiotics alone (antibiotic group) and 39 with a combination of antibiotics and surgery (surgery group). Episodes were regarded as new rather than recurrent if they occurred more than 12 months after the last treated episode; in our three patients who were included twice, the second episode occurred three to five years after the last episode.

At diagnosis of prosthetic valve endocarditis, the mean (SD) age of the subjects was 52 (14) years (range 16 to 76 years). Clinical data were obtained from a review of the patients' medical records. The data included clinical presentation and course, laboratory data, results of microbiological tests, echocardiographic findings, duration of antibiotic treatment, time and type of surgery, and type and location of prosthesis. All operations were performed at our hospital in the division for cardiovascular surgery.

\section{DEFINITIONS}

Patients with mechanical or bioprosthetic valves in the aortic or mitral position were included if they fulfilled the Duke criteria for definite prosthetic valve endocarditis published in detail elsewhere. ${ }^{12}$ The pathological criteria include either demonstrable microorganisms or pathological lesions compatible with active endocarditis. For a clinical diagnosis of endocarditis, two major criteria, one major and three minor criteria, or five minor criteria were needed. Major criteria were: positive blood cultures (typical microorganismus or persistently positive blood cultures) and evidence of endocardial involvement (positive echocardiogram for infective endocarditis or new valvar regurgitation). Minor criteria were: predisposing heart condition or intravenous drug use, fever, vascular phenomena, immunological phenomena, microbiological evidence, and echocardiographic criteria not fulfilling the major criteria as described. ${ }^{11}$

Early prosthetic valve endocarditis was defined as occurring $\leqslant 60$ days after valve replacement, and late prosthetic valve endocarditis as occurring after $>60$ days.

Emergency surgery was defined as valve replacement performed within 24 hours after hospital admission. Early mortality was defined as death within the first 30 days after surgery or death during the hospital stay for patients in the antibiotic group.

Aortoventricular dehiscence was defined as lack of normal continuity of the muscular left ventricular outflow tract with the sinus portion of the aorta caused by abscess formation involving either part of or the whole circumference of the aortic annulus.

\section{ECHOCARDIOGRAPHY}

A complete cross sectional and Doppler transthoracic echocardiographic examination was performed in all patients, with detailed examination of each valve. Transoesophageal echocardiography was routinely available in our hospital from 1991 and was performed in 28 patients $(49 \%)$ between 1991 and 1997 (biplane $5 \mathrm{MHz}$ probe or multiplane $5 \mathrm{MHz}$ probe since 1997). Hewlett-Packard systems (Sonos 1000 and Sonos 2500; HewlettPackard Co, Andover, Massachusetts, USA) were used.

\section{FOLLOW UP}

The patients surviving this hospital admission or their physicians were contacted with a questionnaire to obtain follow up data. These included death, recurrence of prosthetic valve endocarditis, and reoperations. Five patients were interviewed by telephone because the questionnaire was not returned. Follow up was achieved in all patients included in the analysis.

STATISTICAL ANALYSIS

All analyses were performed using the StatView 4.51 statistical software package (Abacus Concepts). Nominal data were analysed using Fisher's exact test. Continuous variables were analysed by the Mann-Whitney test and are presented as mean (SD). Long term follow up was estimated by the KaplanMeier method and compared by the log-rank test. A probability ( $p$ value) $\leqslant 0.05$ was considered significant.

\section{Results}

\section{CLINICAL CHARACTERISTICS}

A comparison of the clinical characteristics of the antibiotic group and the surgery group is shown in table 1 . No significant difference was found between the two methods of treatment with respect to age, sex, concomitant heart disease, number of previous heart operations, history of previous endocarditis, onset of endocarditis (early or late), type of prosthesis, position involved (aortic or mitral), or signs for peripheral emboli. Heart failure and catecholamine requirement were comparable in the two groups. Endocarditis occurred at an average of 57 (69) months (range one to 248 months) after the last valve replacement in the surgery group and at 61 (60) months (range one to 192) in the antibiotic group (NS). The mean time from the onset of clinical symptoms to diagnosis was 20 (22) days (range one to 84) and 14 (25) days (range one to 84 ) in the surgery and antibiotic groups, respectively (NS). 
Table 2 Laboratory results and responsible microorganisms

\begin{tabular}{llll}
\hline Test & $\begin{array}{l}\text { Surgery group } \\
(n=39)\end{array}$ & $\begin{array}{l}\text { Antibiotic group } \\
(n=10)\end{array}$ & $p$ Value \\
\hline ESR $(\mathrm{mm}$ in the first hour $)$ & $57(32)$ & $42(28)$ & $\mathrm{NS}$ \\
WBC $\left(\times 10^{3} / \mu \mathrm{l}\right)$ & $11.6(4.6)$ & $8.4(3.1)$ & 0.047 \\
$\mathrm{CRP}(\mathrm{mg} / \mathrm{l})$ & $133(107)$ & $47(35)$ & 0.02 \\
Early PVE & 4 & 2 & $\mathrm{NS}$ \\
Culture positive & 29 & 8 & $\mathrm{NS}$ \\
Staphylococcus spp & 16 & 1 & 0.048 \\
$\quad$ S aureus & 4 & 0 & $\mathrm{NS}$ \\
$\quad$ Coagulase negative & 12 & 1 & $\mathrm{NS}$ \\
Streptococcus $\mathrm{spp}$ & 7 & 2 & 0.005 \\
Enterococcus spp & 1 & 4 & $\mathrm{NS}$ \\
Other & 5 & 1 & \\
\hline
\end{tabular}

Values are mean (SD) or $\mathrm{n}$.

$\mathrm{CRP}, \mathrm{C}$ reactive protein; ESR, erythrocyte sedimentation rate; PVE, prosthetic valve endocarditis; WBC, white blood cell count.

Table 3 Echocardiographic findings of the surgery group and the antibiotic group

\begin{tabular}{llll}
\hline & $\begin{array}{l}\text { Surgery group } \\
(n=39)\end{array}$ & $\begin{array}{l}\text { Antibiotic group } \\
(n=10)\end{array}$ & $p$ Value \\
\hline Type of valve & 26 & 7 & NS \\
$\quad$ Mechanical & 13 & 3 & $\mathrm{NS}$ \\
$\quad$ Biological & 35 & 8 & \\
Localisation of PVE & 4 & 2 & $<0.0001$ \\
$\quad$ Aortic & 4 & 0 & $<0.0001$ \\
$\quad$ Mitral & 32 & 1 & 0.02 \\
$\quad$ Both & 32 & 1 & $\mathrm{NS}$ \\
Perivalvar leakage & 15 & 0 & \\
Abscess & 9 & 3 & \\
Vortoventricular dehiscence & & & \\
\hline PVE & & &
\end{tabular}

PVE, prosthetic valve endocarditis.

LABORATORY RESULTS AND MICROBIOLOGY

The laboratory findings and the microorganisms causing the endocarditis are shown in table 2 . There was a significantly higher white blood cell count and plasma $C$ reactive protein concentration in the surgery group $(p=0.05$ and $\mathrm{p}=0.02$, respectively). All but three patients (94\%) had antibiotic treatment before being transferred to our hospital. The frequency of culture positive prosthetic valve endocarditis was similar in the two groups. In culture positive patients, staphylococcal infections ( $S$ aureus and coagulase negative staphylococci) were more common in the surgery group ( $p=0.05)$, whereas enterococcal infections were more common in the antibiotic group ( $p=0.005)$. In one blood culture negative patient, histopathological examination identified a possible organism (a Gram negative bacillus). In only one patient, with previous infective endocarditis caused by coagulase negative staphylococci, was the same organism cultured in both episodes.

ECHOCARDIOGRAPHIC AND INTRAOPERATIVE FINDINGS

Echocardiographic and intraoperative findings are listed in table 3. Abscess formation, perivalvar leakage, and aortoventricular dehiscence were more commonly detected in the surgery group $(\mathrm{p}<0.05)$. One patient with enterococcal aortic prosthetic valve endocarditis and an annular abscess seen by transoesophageal echocardiography (maximum diameter $1.5 \mathrm{~cm}$ ) was successfully treated with antibiotics alone, with reduction in the abscess cavity size to less than $1 \mathrm{~cm}$. Echocardiographically detectable abscesses were confirmed surgically in 32 of 33 cases $(97 \%)$, with a positive predictive value of $100 \%$ (95\% confidence interval (CI), $89 \%$ to $100 \%$ ) and a negative predictive value of $86 \%(95 \%$ CI, $42 \%$ to $100 \%)$. Echocardiographically detectable aortoventricular dehiscence was found in 15 of 22 cases $(68 \%)$, which were all confirmed surgically, with a positive predictive value $100 \%$ (95\% CI, $78 \%$ to $100 \%)$ and a negative predictive value $71 \%$ (95\% CI, $49 \% 87 \%$ ).

\section{TREATMENT}

Surgery was performed in 39 patients. The total length of antibiotic treatment was significantly shorter in the surgery group than in the antibiotic group (table $1 ; \mathrm{p}=0.039$ ). In 32 patients $(82 \%)$, elective surgery was possible after 35 (43) days of antibiotic treatment (range 28 to 63). Emergency surgery was necessary in seven patients (18\%) because of an unstable haemodynamic condition; in these patients, congestive heart failure $(p=0.004)$ and aortoventricular dehiscence $(\mathrm{p}=0.005)$ occurred significantly more often than in patients with an elective operation. No significant difference was seen in relation to the type of prosthesis, abscess formation, or staphylococcal infection between the emergency and elective surgery groups (NS). Seven patients $(18 \%)$ in the surgery group died during their hospital admission. One patient died intraoperatively from intractable bleeding and heart failure. Five other patients died within the first 30 days after operation because of multiorgan failure from continuing septicaemia, acute myocardial infarction, retroperitoneal bleeding, or intracerebral haemorrhage. Another patient in the surgery group with severe hypoxic brain damage from preoperative resuscitation died from pneumonia on day 135 (late in-hospital death). Aortoventricular dehiscence $(p=0.05)$, but not congestive heart failure, abscess formation, or staphylococcal infection, was significantly associated with death after surgery.

No patient in the antibiotic group died during the initial hospital admission for endocarditis. However, the difference in early mortality between the surgery and antibiotic groups was not significant ( $0 \%$ v 15\%; NS).

\section{LONG TERM FOLLOW UP}

Follow up was obtained in all patients after 42 (30) months. Length of follow up (41 (32) $v 45$ (24) months) was not significantly different between the surgery and the antibiotic group.

Recurrence of endocarditis occurred in three patients $(9 \%)$ in the surgery group after 46,48 , and 49 months, respectively. All three patients had mechanical valves in the aortic position and reoperation was necessary because of valve dysfunction. Two other patients $(6 \%)$ in the surgery group needed reoperation for dysfunction of the prosthetic valve without signs of recurrent endocarditis. Recurrence of endocarditis also occurred in one patient from the antibiotic group 39 months after a conservatively treated episode of coagulase negative staphylococcal endocarditis (table 4, patient 10). This patient with recurrent coagulase negative staphylococcal endocarditis needed reoperation for congestive heart failure and perivalvar 
Table 4 Clinical, echocardiographic, and microbiological data of the 10 patients treated with antibiotics only

\begin{tabular}{|c|c|c|c|c|c|c|c|c|c|c|c|c|c|}
\hline \multirow[b]{2}{*}{ Patient } & \multirow{2}{*}{$\begin{array}{l}\text { Age } \\
\text { (years) }\end{array}$} & \multirow[b]{2}{*}{ Sex } & \multirow[b]{2}{*}{ Prosthesis } & \multirow[b]{2}{*}{ Localisation } & \multirow{2}{*}{$\begin{array}{l}\text { Previous } \\
\text { endocarditis }\end{array}$} & \multirow{2}{*}{$\begin{array}{l}\text { Heart } \\
\text { failure }\end{array}$} & \multirow[b]{2}{*}{ Emboli } & \multirow[b]{2}{*}{ Microorganism } & \multicolumn{3}{|c|}{ Complications } & \multirow{2}{*}{$\begin{array}{l}\text { Duration of } \\
\text { treatment } \\
\text { (days) }\end{array}$} & \multirow{2}{*}{$\begin{array}{l}\text { Follow up } \\
\text { (months) }\end{array}$} \\
\hline & & & & & & & & & Abscess & AV dehis & Leakage & & \\
\hline 1 & 49 & $M$ & Mech & Aortic & No & No & No & Negative & No & No & No & 312 & 51 \\
\hline 2 & 68 & M & Mech & Mitral & Yes & No & No & Enterococcus spp & No & No & Yes & 132 & 26 \\
\hline 3 & 62 & M & Mech & Mitral & No & No & Yes & Negative & No & No & No & 70 & 54 \\
\hline 4 & 47 & $M$ & Mech & Aortic & No & No & No & Enterococcus spp & Yes & No & No & 42 & 60 \\
\hline 5 & 70 & $M$ & Mech & Aortic & No & No & Yes & Enterococcus spp & No & No & No & 56 & 59 \\
\hline 6 & 50 & $M$ & Mech & Aortic & No & No & Yes & Rothia dentocariosa & No & No & No & 56 & 32 \\
\hline 7 & 72 & $\mathrm{~F}$ & Bio & Aortic & No & No & Yes & Strep sanguis & No & No & No & 42 & 5 \\
\hline 8 & 57 & $M$ & Bio & Aortic & No & No & No & Enterococcus spp & No & No & No & 42 & 91 \\
\hline 9 & 50 & M & Bio & Aortic & No & No & Yes & Strep sanguis & No & No & No & 42 & 36 \\
\hline 10 & 67 & $M$ & Mech & Aortic & Yes & No & No & CNS & No & No & No & 222 & 34 \\
\hline
\end{tabular}

AV dehis, aortoventricular dehiscence; Bio, bioprosthesis; CNS, coagulase negative staphylococcus; Mech, mechanical prosthesis.

leakage. The patient died one hour postoperatively from heart failure. No additional reoperation was needed in the other patients in the antibiotic group. Apart from the above mentioned patient with recurrent staphylococcal endocarditis, the other nine conservatively treated patients had no signs of recurrent infection and good function of the implanted valves after a follow up of 45 (24) months (range five to 91) (table 4). In our analysis, the five year rates for recurrence of prosthetic valve endocarditis were $16 \%$ in the antibiotic group and $14 \%$ in the surgery group (NS). The cumulative five year rate of freedom from recurrent prosthetic valve endocarditis was $85 \%$. The five year rates for need for reoperation were $19 \%$ in the antibiotic and $14 \%$ in the surgery group (NS).

In the surgery group, one patient had sudden cardiac death six years after prosthetic valve endocarditis without signs of recurrent endocarditis. This death was therefore considered to be unrelated to the previous endocarditis. Another two patients died from causes not related to prosthetic valve endocarditis: one had pneumonia four years after the episode of endocarditis and one committed suicide. Overall, neither late mortality ( $18 \%$ v $14 \%$; fig 1$)$ nor the five year rate of event related late mortality $(3 \% \vee 14 \%)$ differed significantly between the antibiotic and the surgery group.

The cumulative five year rates for all our patients with prosthetic valve endocarditis were $83 \%$ survival, $85 \%$ freedom of recurrence, $82 \%$ freedom of reoperation, and $68 \%$ with no event in this period.

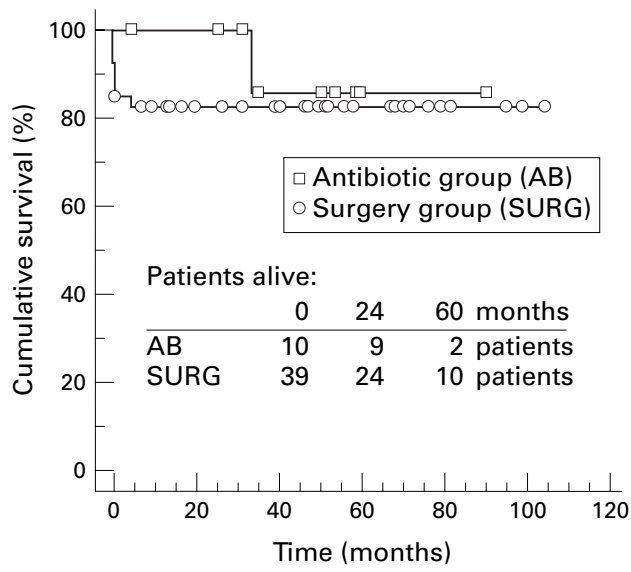

Figure 1 Kaplan-Meier plot for overall survival.
Discussion

Despite improvements in diagnosis and treatment, prosthetic valve endocarditis remains a serious complication associated with a high mortality. In general, combined medical and surgical treatment is considered the management of choice in patients with prosthetic valve endocarditis. The superiority of surgical treatment compared with antibiotic treatment alone has been demonstrated in several studies, and an aggressive approach recommended. ${ }^{110} 1113$ In a large analysis by Calderwood et al, the only variable significantly associated with a worse outcome was initial medical treatment. ${ }^{1}$ In some patients with prosthetic valve endocarditis, severe complications clearly require prompt surgical intervention but only few investigators have analysed which patients can be treated safely by antibiotics alone. ${ }^{14}$

Among the 49 episodes with definite prosthetic valve endocarditis in our study, $10(20 \%)$ were treated conservatively. Our study shows that conservative management of prosthetic valve endocarditis guided by echocardiography and clinical data is feasible for well selected patients without an increase in overall mortality, recurrent endocarditis, and reoperation rate.

CHARACTERISTICS OF PROSTHETIC VALVE ENDOCARDITIS IN OUR STUDY GROUP In our population, $10 \%$ of cases of prosthetic valve endocarditis occurred within the first 60 days after heart valve replacement. This differs from previous reports where approximately one third of the cases were early and two thirds late. ${ }^{15}$ This may reflect the fact that we included only patients with definite prosthetic valve endocarditis and not those with only bacteraemia and no other major criteria of endocarditis, which may be more common early after surgery. In contrast to other reports, ${ }^{71016}$ early prosthetic valve endocarditis in our population was not associated with worse outcome than late endocarditis. The overall frequency of complications like abscess formation (69\%), perivalvar leakage $(67 \%)$, and aortoventricular dehiscence $(45 \%)$ present in our study was similar to that in other published reports. ${ }^{4} 5$ 17-19 In the antibiotic group, however, no patient had aortoventricular dehiscence and only one had an annular abscess and another a perivalvar leakage. The number of previous heart operations was comparable in both groups, although eight patients in the antibiotic group 
$(80 \%)$ had two to three previous operations, compared with $18(46 \%)$ in the surgery group (NS). This might reflect a reluctance to reoperate on patients with two or more previous heart operations. The type of valve, location of the endocarditis, and the presence of vegetations and emboli had a similar frequency in the two groups.

INFLUENCE OF BACTERIOLOGICAL FINDINGS

Early prosthetic valve endocarditis is caused by contamination of the artificial valve at the time of its implantation or by perioperative bacteraemia. Staphylococci ( $S$ aureus and coagulase negative staphylococci) and enterococci are common in early prosthetic valve endocarditis. ${ }^{7}$ Late prosthetic valve endocarditis can be caused by many different microorganisms. ${ }^{13}$ Staphylococci are more prevalent in both early and late prosthetic valve endocarditis compared with endocarditis of the native valves. ${ }^{4}$ The type of microorganism involved is affected by the quantity of potentially infective organisms present, by their special adhesion mechanisms, and by the host environment (for example, whether there is immunosuppression).$^{20} \mathrm{In}$ our study group, in the 37 patients with culture positive endocarditis, staphylococci were the most common organisms, with a prevalence of $46 \%$ and no difference between early and late involvement.

It has been said that the type of microorganism seems to influence the therapeutic strategy, with infections by staphylococci, fungi, and Gram negative bacilli more often requiring a surgical approach. ${ }^{79}$ This was confirmed in our study: all our patients with staphylococcal prosthetic valve endocarditis ultimately needed surgery. Conservative management of staphylococcal endocarditis of prosthetic valves therefore does not seem advisable; there appears to be only a single case report of successful conservative treatment, with a follow up of only one year. ${ }^{21}$ Our conservatively treated patient with coagulase negative staphylococcal endocarditis had a recurrence after 39 months, possibly with the same microorganism despite initial antibiotic treatment for 212 days. In one analysis, ${ }^{22}$ mortality was not influenced by the type of organism but was high, at $41 \%$ for the patients treated with antibiotics only, and no recommendations were given for identifying patients who could be treated conservatively. In a another study involving 122 patients with prosthetic valve endocarditis, ${ }^{23}$ better survival was found for those with combined medical and surgical treatment than with antibiotic treatment alone ( $45 \% v 0 \%)$, and none of 20 patients with $S$ aureus associated endocarditis survived when treated with antibiotics alone. However, in that analysis only subjects who were admitted to the intensive care unit were included and so there was a bias towards very sick patients. When prosthetic valve endocarditis was not caused by staphylococci, the mortality was similar in patients with combined treatment and with antibiotics only treatment $(89 \% v 81 \%)$ in a four month follow up. $^{23}$ The conclusions of John et al that performing valve replacement surgery will reduce mortality among patients with $S$ aureus endocarditis even in the absence of cardiac complications may well be true. ${ }^{24}$ Why were staphylococcal infections more devastating than, for example, enterococcal infections, which had a relatively benign course in our study? It has been observed by others ${ }^{25}$ that enterococcal prosthetic valve endocarditis has a low mortality, $69 \%$ being cured without surgical intervention. This differs completely from enterococcal endocarditis of the native valve, which seems to be very aggressive $e^{25}$; the prosthetic valve appears more resistant to destruction. However, despite the common problem of resistance of enterococci to penicillins, bactericidal treatment with combined aminoglycosides and penicillins for at least six weeks is now a well established way of managing enterococcal native and prosthetic valve endocarditis. In contrast, antibiotic treatment of staphylococcal endocarditis-particularly infections with coagulase negative staphylococci-is not often successful, and this has been confirmed both in vitro and in animal models. $^{26}$

RECURRENCE OF PROSTHETIC VALVE ENDOCARDITIS

Device related infections are a major clinical issue. Infection in a mechanical valve is located in the sewing ring; therefore antibiotic treatment often fails unless the infected implant is removed. ${ }^{13}$ Normally recurrent episodes of endocarditis are defined as relapses occurring within one year of completion of treatment for the initial episode, or as reinfection if they occur more than one year after completion of therapy. In our patients, however, recurrence with possibly the same microorganism (a coagulase negative staphylococcus) was seen to occur as late as 39 months after valve replacement. We propose, therefore, that recurrence of prosthetic valve endocarditis is defined as any episode of endocarditis occurring with the same organism, even during long term follow up. Only if the organism differs should reinfection be postulated. It was worrying that in all four patients with recurrent endocarditis, this occurred very late in the follow up (39 to 49 months), which could indicate that in such cases it is difficult to be sure of a cure. However, the risk of recurrence or persistent sepsis or infection is low. ${ }^{19}$

\section{REOPERATION AND MORTALITY}

Early mortality understandably tended to be higher in the surgery group $(15 \%)$ than in the antibiotic group $(0 \%)$, though this difference was not significant $(p=0.15)$. This was because the sicker patients were treated surgically. We were, however, especially interested in the long term follow up data. In a cumulative five year rate, freedom from recurrence was $85 \%$ in all our patients after discharge from the initial hospital stay. The five year rate for recurrent endocarditis was not significantly different in the two groups. We had an excellent overall $83 \%$ cumulative five year survival rate at follow up. Our late mortality was $14 \%$ in the antibiotic group and 
$18 \%$ in the surgery group (NS). Studies published before 1980 reported mortality rates for early and late prosthetic valve endocarditis of $70 \%$ and $45 \%$, respectively. ${ }^{25}$ More recent studies on the surgical treatment of prosthetic valve endocarditis report an overall in-hospital mortality of approximately $10-25 \%{ }^{121} 252728$ and a five year survival ranging from $50 \%$ to $75 \%$. The cumulative five year survival rate of $83 \%$ in our study group was therefore better than expected. The cumulative five year rate of freedom from any event was $68 \%$. The rates of reoperation for recurrent endocarditis and for late sequelae were also lower in our analysis than in other studies.

In certain patient groups, conservative treatment can thus result in comparable survival to surgical treatment. We chose to perform reoperations on the basis of the following criteria: congestive heart failure from valve dysfunction, recurrent emboli despite treatment, and progression of cardiac complications such as large abscess formation or aortoventricular dehiscence. Early non-staphylococcal prosthetic valve endocarditis as such was not a criterion for reoperation, in contrast to other recommendations. ${ }^{8}$

IMPACT OF ECHOCARDIOGRAPHY

The important role of echocardiography in diagnosis and management of prosthetic valve endocarditis has been emphasised. ${ }^{829} 30$ Echocardiographic signs of prosthetic valve endocarditis include vegetations on and around the prosthetic valve, valve dysfunction (stenosis, regurgitation, paravalvar leak), and invasion of perivalvar tissue by abscesses and fistulae. ${ }^{8}$ Early diagnosis of prosthetic valve endocarditis-especially in the presence of negative blood cultures caused by previous use of antibiotics - is thus facilitated by the use of transthoracic, and if this is inconclusive transoesophageal, echocardiography. ${ }^{30}$ Vegetations are known to occur in $68-86 \%$, and annular or ring abscesses in 35-100\%; abscess formation is less common in mitral prosthetic valve endocarditis, with an incidence of $23-35 \%$, compared with up to $83 \%$ in aortic prosthetic valve endocarditis. ${ }^{30}$ In our patient group, abscesses were detected overall in 33 patients $(67 \%)$ and perivalvar leakage in 33 patients (67\%). Vegetations were only seen in 12 patients $(24 \%)$. This proportion is lower than that reported elsewhere, probably because we had only used routine transoesophageal echocardiography since 1991 (in all in 28 patients $(57 \%)$ ) and might therefore have missed some vegetations in conservatively treated patients. It has been shown by Lengyel that the sensitivity of detecting abscess formation is much lower by transthoracic echocardiography than by transoesophageal echocardiography: four cases $v 14$ in a group of 31 patients. $^{30}$ Lengyel also showed that with early detection of complications by transoesophageal echocardiography, mortality can be reduced. One of our patients with aortic enterococcal endocarditis had an abscess treated successfully with antibiotics alone; the abscess cavity became smaller and there were no signs of aortoventricular dehis- cence. Echocardiographic findings of abscess formation and aortoventricular dehiscence in our patients were all confirmed at surgery (positive predictive value $100 \%$ ), with acceptable negative predictive values of $86 \%$ and $71 \%$, respectively. Echocardiography is the most important tool for treatment decisions in prosthetic valve endocarditis: in clinically unstable patients who need surgery anyway, it is necessary in order to describe the extent of the endocarditis; in stable patients it might help in deciding who can be treated conservatively. In patients without abscess formation, or with the rare resolution of small abscess cavities and no aortoventricular dehiscence, conservative treatment may be attempted for nonstaphylococcal prosthetic valve endocarditis. Paramount to overall surgical success is a well defined preoperative strategy, for which an echocardiographic definition of the presence and extent of significant complications is important. ${ }^{24}$

\section{STUDY LIMITATIONS}

Our study population was small, with 46 patients and 49 episodes of endocarditis. However, we analysed only patients who fulfilled the Dukes criteria for definite endocarditis as mentioned above, and these criteria have been shown to have a high sensitivity and a high negative predictive value. ${ }^{28-33}$ Previous studies have not had such stringent criteria-for example, in the multicentre trial by Yu et al only 42 of the 74 patients had definite endocarditis. ${ }^{10}$ The study of Kuyvenhoven et al included 70 episodes of prosthetic valve endocarditis but only 39 definite episodes. ${ }^{27}$ Thus our study sample of patients with definite prosthetic valve endocarditis was at least of comparable size and involved only definite cases. These were therefore more severe than the average found in other studies.

Decisions about the therapeutic approach were left entirely to the treating physician and were not prospectively randomised. Therefore, we cannot definitely identify particular features that could allow us to make general recommendations for the safe conservative treatment of prosthetic valve endocarditis.

\section{CONCLUSIONS}

Patients with non-staphylococcal prosthetic valve endocarditis without left heart failure and with careful clinical and echocardiographic surveillance to exclude large abscesses or aortoventricular dehiscence may be managed by antimicrobial treatment alone without an increased rate of reinfection, reoperation, or death.

This study was presented as an oral communication at the American College of Cardiology Meeting 1999 in New Orleans, USA, March 1999.

1 Calderwood SB, Swinski LA, Karchmer AW, et al. Prosthetic valve endocarditis. Analysis of factors affecting outcome of therapy. $\mathcal{F}$ Thorac Cardiovasc Surg 1986;92:776-83.

2 Horstkotte D, Schulte HD, Bricks W, et al. Prosthetic valve endocarditis: incidence, diagnosis, therapeutic decisions, endocarditis: incidence, diagnosis, therapeutic decisions, and prognosis. Schweiz Med Woschenschr 1987;117.1671-8. Douglas PS, Hirshfeld JW, Edie RN, et al. Clinical comparison of St Jude and porcine aortic value prostheses. Circula-
tion 1985;72:II-135-9. 
4 Berlin JA, Abrutyn E, Strom BL, et al. Incidence of endocarditis in the Delaware Valley 1988-90. Am f Cardio $1995 ; 76: 933-6$.

5 Binder T, Baumgartner H, Maurer G. Diagnosis and management of prosthetic valve dysfunction. Curr Opin Cardiol 1996;11:131-8.

6 Stanbridge TN, Isalska BJ. Aspects of prosthetic valve endocarditis. F Infect 1997;35:1-6.

7 Wilson WR, Danielson GK, Giuliani ER, et al. Prosthetic valve endocarditis. Mayo Clin Proc 1982;57:155-61.

8 Vlessis AA, Khanki A, Grunkemeier GL, et al. Risk, diagnosis and management of prosthetic valve endocarditis: a review. F Heart Valve Dis 1997;6:443-65.

9 Karchmer AW, Bisno AL. Infections of prosthetic heart valves and vascular grafts. In: Bisno AL, Waldvogel FA, eds. Infections associated with indwelling medical devices. Washington DC: American Society of Microbiology 1989:129-59.

$10 \mathrm{Yu}$ VL, Fang GD, Keys TF, et al. Prosthetic valve endocarditis: superiority of surgical valve replacement versus medical therapy only. Ann Thorac Surg 1994;58:1073sus

11 Rocchiccioli C, Chastre J, Lecompte Y, et al. Prosthetic valve endocarditis: the case for prompt surgical manage-

12 Durack DT, Lukes AS, Bright DK, and the Duke Endocarditis Service. New criteria for diagnosis of infective endocarditis: utilization of specific echocardiographic findings. Am F Med 1994;96:200-9.

13 David TE. The surgical treatment of patients with prosthetic valve endocarditis. Semin Thorac Cardiovasc Surg 1995;7:47-53.

14 Karchmer AW, Dismukes WE, Buckley MJ, et al. Late prosthetic valve endocarditis: clinical features influencing therapy Am F Med 1978;64:199-206.

15 Threkeld MG, Cobbs CG. Infectious disorders of prosthetic valves and intravascular devices. In: Mandell GL, Bennett JE, Dolin R, eds.Principles and practice of infectious diseases. New York: Churchill Livingstone, 1995: 783-93.

16 Dismukes WE, Karchmer AW, Buckley MJ, et al. Prosthetic valve endocarditis: analysis of 38 cases. Circulation 1973;48: valve enci $365-77$.

17 Tornos P, Almirante B, Olona M, et al. Clinical outcome and long-term prognosis of later prosthetic valve endocarditis: a 20-year experience. Clin Infect Dis 1997;24: $381-6$

18 Daniel WT, Mugge A, Grote J, et al. Comparison of transthoracic and transesophageal echocardiography for detection of abnormalities of prosthetic and bioprosthetic valves in the mitral and aortic positions. Am $\mathcal{F}$ Cardio 1993;71:210-15.
19 Pansini S, di Summa M, Patane F, et al. Risk of recurrence after reoperation for prosthetic valve endocarditis. $\mathcal{F}$ Heart Valve Dis 1997;6:84-7.

20 Horstkotte D, Weist K, Rüden H. Better understanding of the pathogenesis of prosthetic valve endocarditis - recent perspectives for prevention strategies. F Heart Valve Dis 1998;7:313-15.

21 Bruss J, Jacobs LE, Kotler MN, et al. Utility of transesophageal echocardiography in the conservative management of prosthetic valve endocarditis. Chest 1992;102:1886-8.

22 Grover FL, Cohen DJ, Oprian C, et al. Determinants of the occurrence of and survival from prosthetic valve endocarditis. $\mathcal{F}$ Thorac Cardiovasc Surg 1994;108:207-14.

23 Wolff $\mathrm{M}$, Witchitz S, Chastang C, et al. Prosthetic valve endocarditis in the ICU. Prognostic factors of overall survival in a series of 122 cases and consequences for treatment decision. Chest 1995;108:688-94.

24 John MDV, Hibberd PL, Karchmer AW, et al. Staphylococcus aureus prosthetic valve endocarditis: optimal management and risk factors for death. Clin Infect Dis 1998;26: 1302-9.

25 Rice LB, Calderwood B, Eliopoulos GM, et al. Enterococcal endocarditis: a comparison of prosthetic and native valve disease. Rev Infect Dis 1991;13:1-7.

26 Schwank S, Rajacic Z, Zimmerli W, et al. Impact of bacterial biofilm formation on in vitro and in vivo activities of antibiotics. Antimicrob Agents Chemother 1998;42:895-8.

27 Kuyvenhoven JP, van Rijk-Zwikker GL, Hermans J, et al. Prosthetic valve endocarditis: analysis of risk factors for mortality. Eur 7 Cardiothorac Surg 1994;8:420-4.

28 Jault F, Gandibakhch I, Chastre JC, et al. Prosthetic valve endocarditis with ring abscesses. F Thorac Cardiovasc Surg endocarditis with ring

29 Bruss J, Jacobs LE, Kotler MN, et al. Utility of transesophageal echocardiography in the conservative management of prosthetic valve endocarditis. Chest 1992;102:1886-8.

30 Lengyel $M$. The impact of transesophageal echocardiography on the management of prosthetic valve endocarditis: experience of 31 cases and review of the literature. F Heart Valve Dis 1997;6:204-11.

31 Wilson WR, Danielson GK, Giulani ER, et al. Prosthetic valve endocarditis. Mayo Clin Proc 1982;57:155-61.

32 Gordon SM, Keys TF. Bloodstream infections in patients with implanted prosthetic cardiac valves. Semin Thorac Cardiovasc Surg 1995;7:2-6.

33 Stewart WJ, Shan K. The diagnosis of prosthetic valve endocarditis by echocardiography. Semin Thorac Cardiovasc Surg 1995;7:7-12 